$8^{\text {th }}$ International conference on Research in Engineering, Science and Technology Paris, France November 2-4, 2018

\title{
Natural convection characteristics in a vertical open channel with partially heated surfaces
}

\author{
Eunpil Kim \\ Pukyong National University
}

\begin{abstract}
This paper presents open channel natural convection with separated boundary conditions. To find natural convection characteristics the channel aspect ratio (pitch verse height) and wall boundary aspect ratio (first-type boundary verse second-type boundary aspect) are investigated between two vertical parallel plates. The convective heat transfer characteristics are evaluated numerically while the channel inlet is kept at a fully developed velocity profile and constant temperature. Steady-state two-dimensional laminar natural convection is analyzed. Computational procedure is selected by control volume method with pressure treatment of body force weighted method. The comparison numerical results are in good agreement with experiment results. Results show that when the channel ratio decreases, the heated area deceases because mass flow rate increases. The Nusselt values linearly increase with increasing isothermal wall length.
\end{abstract}


